\title{
EXISTENCE, UNIQUENESS AND REGULARITY OF SOLUTIONS TO A CLASS OF THIRD-KIND VOLTERRA INTEGRAL EQUATIONS
}

\author{
SONIA SEYED ALLAEI, ZHAN-WEN YANG AND HERMANN BRUNNER
}

Communicated by Giovanni Monegato

\begin{abstract}
We analyze the existence, uniqueness and regularity of solutions to a class of third-kind Volterra integral equations, including equations with weakly singular kernels. Of particular interest are those integral equations that can be transformed into cordial Volterra integral equations whose underlying integral operator may be non-compact.
\end{abstract}

1. Introduction. We study the existence and regularity of solutions to the linear third-kind Volterra integral equation

$$
t^{\beta} u(t)=f(t)+\int_{0}^{t}(t-x)^{-\alpha} k(t, x) u(x) d x, \quad t \in I:=[0, T],
$$

where $\alpha \in(0,1)$ and $\beta>0$. In (1.1), $f$ and $k$ are given continuous realvalued functions on $I$ and $\Delta:=\{(t, x): 0 \leq x \leq t \leq T\}$, respectively.

Our aim is to establish conditions under which equation (1.1) possesses a unique solution $u \in C(I)$. Our analysis is based on the fact that (1.1) can be rewritten as a so-called cordial Volterra integral equation (cf., Vainikko $[\mathbf{2 0}, \mathbf{2 1}]$ ), whose underlying integral operator

$$
\left(T_{k, \beta, \alpha} u\right)(t):=\int_{0}^{t} t^{-\beta}(t-x)^{-\alpha} k(t, x) u(x) d x
$$

2010 AMS Mathematics subject classification. Primary 45A05, 45D05, 45E99.

Keywords and phrases. Volterra integral equation of the third kind, cordial Volterra integral equation, existence and regularity of solutions.

The research of the first author was co-financed by the Hong Kong Research Grants Council (RGC project No. 1369648) and a CEMAT grant under the framework of the project PTDC/MAT/101867/2008 funded by Fundação para a Ciência e a Tecnologia (FCT), Portugal. The research of the third author was supported by the Hong Research Grants Council (GRF Grant HKBU 200211).

Received by the editors on December 3, 2014, and in revised form on March 31, 2015. 
is not always a compact operator on $C(I)$.

The notion of a (Fredholm-type) integral equation of the third kind was introduced around 1910, by Picard [16] and by Hilbert [12]. Such equations were later studied, for example, in $[\mathbf{1}, \mathbf{3}, \mathbf{4}, \mathbf{7}, \mathbf{8}, \mathbf{9}, \mathbf{1 0}, \mathbf{1 4}$, 17, 19]. Volterra integral equations of the third kind have received less attention, although the first study was carried out, in 1910 and 1911, by Evans [5, 6] (who spent most of 1910-1912 studying with Volterra in Rome). He analyzed the solvability of the more general form of (1.1),

$$
a(t) u(t)=f(t)+\int_{0}^{t} k(t, x) u(x) d x, \quad t \in I,
$$

where $a(t)$ is continuous and has a finite number of zeros in $I$.

In 1953, Sato [18] studied power series solutions for the nonlinear third-kind Volterra integral equation

$$
x u(x)=f(x)+\int_{0}^{x} K(x, t, u(t)) d t, \quad t \geq 0,
$$

where $f(x)$ and $K(t, x, u)$ are analytic near $(x, t, u)=\left(0,0, c_{0}\right)$ for some $c_{0} \in \mathbb{R}$. In 1977, Fényes [7] considered integral equations of the form (1.2) when $a(t)=t+c(c<0)$ and the kernel is of convolution type. In 1998, Pereverzev and Prössdorf [15] extended the analysis in [19] of the ill-posed nature of third-kind Fredholm integral equations to a very general third-kind Volterra integral equation with weakly singular kernels. More recent studies are due to Imanaliev [13] and Grandits [11] (who analyzed the regularity of solutions).

The aim of this paper is to study the existence, uniqueness and regularity properties of solutions of (1.1) for $\beta \in(0,1]$ and $\beta>1$, both for smooth kernels $(\alpha=0)$ and weakly singular kernels $(0<\alpha<1)$. In particular, we derive conditions on $f$ and $k$ which lead to so-called cordial Volterra integral equations (see Definition 2.1).

2. Preliminaries and definitions. In order to employ the theory of cordial Volterra integral equations we write the given equation (1.1) in the form:

$$
u(t)=t^{-\beta} f(t)+\int_{0}^{t} t^{-\beta}(t-x)^{-\alpha} k(t, x) u(x) d x, \quad t \in(0, T] .
$$


The existence of a solution $u \in C(I)$ to (2.1) will obviously depend on the regularity of $g(t):=t^{-\beta} f(t)$ at $t=0^{+}$, as the following examples illustrate.

Example 2.1. In the third-kind Volterra integral equation,

$$
t^{3 / 2} u(t)=\sqrt{t}(1-2 \sqrt{t+1})+\int_{0}^{t} \sqrt{x(t+1)} u(x) d x, \quad t \in[0,1]
$$

we have $f(0)=0$ and $\beta=3 / 2$. It is easy to verify that, for any continuous function $u \in C[0,1]$, the function given by

$$
u(t)-t^{-3 / 2} \int_{0}^{t} \sqrt{x(t+1)} u(x) d x
$$

is continuous on the closed interval $[0,1]$. But $g(t)=t^{-3 / 2} \sqrt{t}(1-$ $2 \sqrt{t+1})$ is not continuous on $[0,1]$. Hence, this third-kind Volterra integral equation does not have a continuous solution on $[0,1]$.

Example 2.2. The third-kind Volterra integral

$$
t^{2} u(t)=f(t)+\int_{0}^{t}(t-x)^{-1 / 2} x^{3 / 2} u(x) d x, \quad t \in[0,1],
$$

with

$$
f(t)=t^{2}(0.26-0.18 \ln (t))
$$

has $f$ in $C[0,1]$ and $f(0)=0$. Since, for any function $u \in C[0,1]$, the function

$$
u(t)-t^{-2} \int_{0}^{t}(t-x)^{-1 / 2} x^{3 / 2} u(x) d x
$$

is continuous on the interval $[0,1]$, while $g(t):=t^{-2} f(t)$ is not continuous at $t=0$, the above third-kind Volterra integral equation cannot possess a solution $u \in C[0, T]$.

For certain values of $\alpha$ and $\beta$ the equation (2.1) can be viewed as a cordial Volterra integral equation defined by Vainikko [20, 21].

Definition 2.3. The second-kind Volterra equation

$$
\mu u(t)=f(t)+\left(V_{\varphi, a} u\right)(t), \quad t \in I:=[0, T]
$$


(with $\mu \in \mathbb{C}$ ) is called a cordial Volterra integral equation of the second kind if $V_{\varphi, a}$ is of the form:

$$
\left(V_{\varphi, a} u\right)(t):=\int_{0}^{t} t^{-1} \varphi(x / t) a(t, x) u(x) d x, \quad t \in I,
$$

where $a(t, x)$ is at least continuous on $\Delta=\{(t, x): 0 \leq x \leq t \leq T\}$. This integral operator is said to be a cordial Volterra integral operator with core $\varphi$ if $\varphi \in L^{1}(0,1)$.

A cordial Volterra integral operator (2.2) is a bounded linear operator from $C(I)$ to $C(I)$.

Theorem 2.4. [20, 21]. The operator $V_{\varphi, a}$ is compact if and only if $a(0,0)=0$. If $a(0,0) \neq 0, V_{\varphi, a}$ is non-compact (as an operator from $C(I)$ into $C(I))$, and its spectrum is given by

$$
\sigma_{C(I)}\left(V_{\varphi, a}\right)=\{0\} \cup\{a(0,0) \hat{\varphi}(\lambda): \operatorname{Re}(\lambda) \geqslant 0\},
$$

where

$$
\widehat{\varphi}(\lambda):=\int_{0}^{1} \varphi(r) r^{\lambda} d r
$$

Moreover, for any $a \in C^{m}(\Delta)$ with $a(0,0) \neq 0, V_{\varphi, a}$ maps $C^{m}(I)$ to $C^{m}(I)$, and its spectrum is

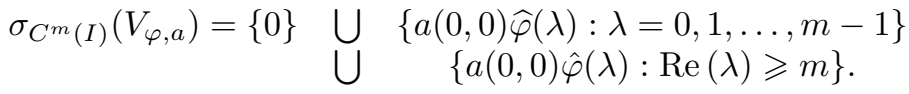

In Section 3, we will present results on the solvability of equation (1.1) or, equivalently, (2.1), when the kernel $k$ is continuous $(\alpha=0)$. The results on the existence and uniqueness of the solution of equation (1.1) for weakly singular kernels $(0<\alpha<1)$ will be established in Section 4.

3. Third-kind Volterra integral equations with continuous kernels. The existence and uniqueness of solutions to (1.1) with continuous kernels are governed by the properties of the integral operator:

$$
\left(T_{k, \beta} u\right)(t):=\int_{0}^{t} t^{-\beta} k(t, x) u(x) d x
$$


they will be seen to depend on the mapping properties (boundedness, compactness or non-compactness) of the Volterra integral operator in (2.1).

3.1. The case $\beta=1$. Here, the integral operator corresponds to a cordial Volterra integral operator with the constant core $\varphi(x) \equiv$ 1. Hence, the following theorem is an immediate consequence of Theorem 2.4.

Theorem 3.1. For a continuous kernel $k \in C(\Delta)$, the operator $T_{k, 1}$ is compact if and only if $k(0,0)=0$. For $k(0,0) \neq 0, T_{k, 1}$ is a noncompact operator, and its spectrum is given by

$$
\sigma_{C(I)}\left(T_{k, 1}\right)=\{0\} \cup\left\{\frac{k(0,0)}{1+\lambda}: \operatorname{Re}(\lambda) \geqslant 0\right\} .
$$

For $k \in C^{m}(\Delta)(m \geq 1)$, the integral operator $T_{k, 1}$ maps $C^{m}(I)$ into $C^{m}(I)$. Its spectrum is

$$
\begin{aligned}
\sigma_{C^{m}(I)}\left(T_{k, 1}\right)=\{0\} & \cup\left\{\frac{k(0,0)}{1+\lambda}: \lambda=0,1, \ldots, m-1\right\} \\
& \left.\cup \frac{k(0,0)}{1+\lambda}: \operatorname{Re}(\lambda) \geqslant m\right\} .
\end{aligned}
$$

Therefore, the existence, uniqueness and regularity of solutions to (1.1) with $\beta=1$ and $\alpha=0$ can be deduced directly from the results in [23]. The following theorem describes the conditions under which the equation (1.1) possesses a unique continuous solution on $I$. We use the notation $g(t):=t^{-1} f(t)$.

Theorem 3.2. Assume that the kernel $k(t, x)$ in the third-kind Volterra integral equation

$$
t u(t)=f(t)+\int_{0}^{t} k(t, x) u(x) d x, \quad t \in I,
$$

satisfies $k \in C(\Delta)$ and $f \in C(I)$ is such that $g \in C(I)$. Then:

(a) If $k(0,0)<1$, there exists a unique continuous solution.

(b) If $k(0,0)=1$, there exists at most one solution.

(c) If $k(0,0)>1$, there exist multiple solutions for all $g$. 
Remark 3.3. When $k(0,0)=1, g(0)=0$ and $g \in C^{1}(I)$, there exists a unique solution $u \in C(I)$. Details can be found in [23].

The following theorem deals with the regularity of solutions to (3.3) ([21]).

Theorem 3.4. If $k \in C^{m}(\Delta)$ and $g \in C^{m}(I)$, then for $k(0,0)<1$ the unique solution $u$ of the equation (3.3) is in $C^{m}(I)$.

Remark 3.5. If $f \in C^{m}(I)$ with $f(0)=0$, then the function $g(t)=t^{-1} f(t)$ lies in $C^{m-1}(I)$. Hence, for $k(0,0)<1$, the unique solution of equation (3.3) belongs to $C^{m-1}(I)$.

3.2. The case $\beta \in(0,1)$. By Theorem 2.4, the Volterra integral operator $(3.1)$ with $\beta \in(0,1)$ is compact, and hence its spectrum is $\{0\}$. The existence and uniqueness of solutions to (1.1) then is guaranteed by the classical theory of second-kind Volterra integral equations (cf., [2, Chapter 2]).

Theorem 3.6. Assume that in the third-kind Volterra integral equation (1.1) with $\alpha=0$ and $0<\beta<1$ the function $f$ is such that $g(t):=$ $t^{-\beta} f(t)$ is continuous on I. If $k \in C(\Delta)$, the equation possesses a unique solution $u \in C(I)$.

The regularity of $u$ for the case $0<\beta<1$ is related to the existence result for the case $\beta>1$, as we will explain in the next section.

3.3. The case $\beta>1$. If $\beta>1$ the integral operator (3.1) is a bounded operator on $C(I)$ if its kernel $k(t, x)$ has the form

$$
k(t, x)=x^{\beta-1} h(t, x),
$$

with $h \in C(\Delta)$. The following example shows that this operator may be unbounded if $k(t, x)$ does not have the above form (3.4).

Example 3.7. The operator

$$
\left(T_{k, \beta} u\right)(t)=\int_{0}^{t} t^{-3} \sqrt{t x} u(x) d x,
$$


maps $u(t) \equiv 1$ to $\left(T_{k, \beta} u\right)(t)=2 /(3 t)$, which is unbounded on $[0,1]$.

Remark 3.8. In this paper, we do not explicitly consider kernels of the form

$$
k(t, x)=t^{\gamma} h(t, x),
$$

with $\gamma \in(0, \beta)$ and $h \in C(\Delta)$ because, in this case, the equation (1.1) can be reduced to the equation

$$
t^{\beta-\gamma} u(t)=t^{-\gamma} f(t)+\int_{0}^{t} h(t, x) u(x) d x,
$$

with $0<\beta-\gamma<\beta$.

Remark 3.9. If we rewrite the integral operator (3.1) as

$$
\left(T_{k, \beta} u\right)(t)=\int_{0}^{1} H(t, r) u(t r) d r,
$$

where $H(t, r)=t^{1-\beta} k(t, t r)$, we readily verify that it is a bounded operator on $C[0, T]$ provided it satisfies the following two conditions:

(a) $H(t, r)$ is integrable with respect to $r$ for each $t \in[0, T]$;

(b) the function

$$
J(t):=\int_{0}^{1} H(t, r) d r
$$

is continuous on $[0, T]$.

In this paper, we only consider kernels of the form (3.4) which satisfy the above conditions.

The part $h(t, x)$ in the kernel defined in (3.4) can be non-continuous with respect to $x$ at zero, but the operator may still be bounded and continuous.

We will describe the solvability of the equation (1.1) when $h \in C(\Delta)$. Results corresponding to a weaker condition on $h(t, x)$ will be presented in a future paper.

Theorem 3.10. For $\beta>1$, the operator $T_{k, \beta}$ defined in (3.1) whose kernel has the form (3.4), with $h \in C(\Delta)$, maps $C(I)$ into $C(I)$ and 
is bounded on $C[0, T]$. The operator is compact when $h(0,0)=0$; otherwise, we have a non-compact operator with the spectrum

$$
\sigma_{C(I)}\left(T_{k, \beta}\right)=\{0\} \cup\left\{\frac{h(0,0)}{\beta+\lambda}: \operatorname{Re}(\lambda) \geqslant 0\right\} .
$$

If, in addition, $h \in C^{m}(\Delta)$, then $T_{k, \beta}$ maps $C^{m}(I)$ to $C^{m}(I)$, and its spectrum is

$$
\begin{aligned}
\sigma_{C^{m}(I)}\left(T_{k, \beta}\right)=\{0\} & \cup\left\{\frac{h(0,0)}{\beta+\lambda}: \lambda=0,1, \ldots, m-1\right\} \\
& \cup\left\{\frac{h(0,0)}{\beta+\lambda}: \operatorname{Re}(\lambda) \geqslant m\right\} .
\end{aligned}
$$

Proof. For $\beta>1$ and the kernel (3.4) with $h(0,0) \neq 0$, the operator (3.1) is a cordial operator, namely,

$$
\left(T_{k, \beta} u\right)(t)=\int_{0}^{t} t^{-1}\left(\frac{x}{t}\right)^{\beta-1} h(t, x) u(x) d x,
$$

since its core $\varphi(x)=x^{\beta-1}$ is in $L^{1}(0,1)$. Thus, the results follow from Theorem 2.4.

In the next theorem, we state sufficient conditions for equation (1.1) with $\alpha=0$ and $\beta>1$ to have a unique continuous solution when $h \in C(\Delta)$. We set $g(t):=t^{-\beta} f(t)$ and assume that $f \in C(I)$ is such that $g \in C(I)$.

Theorem 3.11. Assume that the kernel $k(t, x)$ in the third-kind Volterra integral equation (1.1) is of the form (3.4), with $k, h \in C(\Delta)$. Then:

(a) If $h(0,0)<\beta$, there exists a unique continuous solution.

(b) If $h(0,0)=\beta$, there exists at most one continuous solution.

(c) If $h(0,0)>\beta$, the solution is not unique.

Proof. The form of the spectrum defined in (3.5) implies ([21]) that if $1 \notin \sigma_{C[0, T]}\left(T_{k, \beta}\right)$ (that is, the real-valued function $h(0,0)$ must be less than $\beta$ for $h(0,0) \neq \lambda+\beta$ to hold), the integral equation possesses a unique continuous solution for any $g \in C(I)$. If 1 belongs to the spectrum and $h(0,0) \geq \beta$, it is shown in [23] that, if, in addition, 1 lies on the boundary of the spectrum, we have $h(0,0)=\beta$, and hence case 
(b). Otherwise, [21] shows that the equation has multiple solutions for any $g \in C(I)$.

The following theorem describes the regularity properties of the integral operator $(3.1)$ when $0<\beta<1$.

Theorem 3.12. Let $m \geq 1$ be an integer and $0<\beta<1$. Assume that the continuous kernel

$$
k(t, x)=x^{\beta+m-1} h(t, x)
$$

satisfies the following conditions:

(i) $\frac{\partial^{j}}{\partial t^{j}} k \in C(\Delta)$ for $j=0, \ldots, m$;

(ii) $\frac{\partial^{j}}{\partial t^{j}} h \in C(\Delta)$ for $j=0, \ldots, m-1$;

(iii) the function $H_{j+1}(t):=\frac{\partial^{j}}{\partial t^{j}} h(t, t)$ belongs to $C^{m-j-1}(I)$ for $j=$ $0, \ldots, m-1$.

Then the operator $T_{k, \beta}$ defined in (3.1) is a continuous map from $C^{m-1}(I)$ into $C^{m}(I)$.

Proof. If $u \in C(I)$, we may differentiate $T_{k, \beta} u$, to obtain

$$
\left[\left(T_{k, \beta} u\right)(t)\right]^{\prime}=h(t, t) u(t)-\beta\left(T_{k, \beta+1} u\right)(t)+\left(T_{b, \beta} u\right)(t),
$$

where

$$
b(t, x):=\frac{\partial}{\partial t} k(t, x) .
$$

The conditions imposed on the kernel $k(x, t)$ ensure that the operator $T_{k, \beta+1}$ maps $C(I)$ to $C(I)$. Hence, the result is true for $m=1$. For $m>1$, the $m$ th derivative of $T_{k, \beta} u(t)$ is of the form:

$$
\left(T_{k, \beta} u\right)^{(m)}(t)=F_{m}(t)+\sum_{k=0}^{m} c_{k}^{\prime} \int_{0}^{t} t^{-\beta-k} \frac{\partial^{m-k}}{\partial t^{m-k}} k(t, x) u(x) d x,
$$

where

$$
F_{m}(t)=\sum_{j=1}^{m}\left(\sum_{k=0}^{j-1} c_{j k} t^{-\beta-k} \frac{\partial^{j-1-k}}{\partial t^{j-1-k}} k(t, t) u(t)\right)^{(m-j)},
$$

with $c_{k}^{\prime}(k=0, \ldots, m)$ and $c_{j k}(j=1, \ldots, m ; k=0, \ldots, j-1)$ denoting appropriate constants. The proof is now completed by taking into 
account that all integral operators occurring on the right-hand side of (3.8) are continuous under the conditions of the theorem.

The regularity properties of the solution of the third-kind Volterra integral equation (1.1) with $0<\beta<1$ are described in the following theorem. Recall that $g(t):=t^{-\beta} f(t)$.

Theorem 3.13. Consider the third-kind Volterra integral equation (1.1) with $0<\beta<1$. If $g \in C^{m}(I)$ and the conditions of the Theorem 3.12 hold, the unique solution of (1.1) is in $C^{m}(I)$.

Proof. Theorem 3.6 implies that the unique solution of (1.1) is in $C(I)$. It then follows from Theorem 3.12 and the assumption that $g \in C^{1}(I)$ that $u \in C^{1}(I)$. By induction, the solution can be shown to be in $C^{m}(I)$ if $g \in C^{m}(I)$. This completes the proof.

Remark 3.14. For $f \in C^{m}[0, T]$ with $f(0)=0$, and $\beta \in(0,1)$, the function $g(t)=t^{-\beta} f(t)$ is in general only continuous but not continuously differentiable. So the solution of the equation (1.1) belongs only to $C(I) \backslash C^{1}(I)$.

The regularity properties of the solutions to the third-kind Volterra integral equation corresponding to the operator $T_{k, \beta}$ with $\beta>1$ are described in the following theorem (see also [21]).

Theorem 3.15. Consider the third-kind Volterra integral equation (1.1) with $\beta>1$ and kernel (3.4), and assume that $h \in C^{m}(\Delta)$ and $g \in C^{m}(I)$. Then, if $h(0,0)<\beta$, the unique solution $u \in C(I)$ of (3.3) lies in $C^{m}(I)$.

The following two theorems are about the regularity of the solution for $\beta>1$, but under weaker conditions on the kernel. Their proofs are similar to the ones for Theorems 3.7 and 3.8; we leave them to the reader.

Theorem 3.16. Assume that kernel defining the integral operator $T_{k, \beta}$ (cf., (3.1)) with $\beta>1$ and $\alpha=0$ has the following properties:

(i) The kernel is of the form (3.7) with $k \in C(\Delta)$; 
(ii) $\frac{\partial^{j}}{\partial t^{j}} h \in C(\Delta)$ for $j=0, \ldots, m$;

(iii) $H_{j+1}(t):=\frac{\partial^{j}}{\partial t^{j}} h(t, t)$ is in $C^{m-j-1}(I)$ for $j=0, \ldots, m-1$.

Then the operator is a bounded map from $C^{m-1}[0, T]$ into $C^{m}[0, T]$.

Theorem 3.17. Consider the third-kind Volterra integral equation (1.1), and assume that the conditions of the previous theorem hold. Then for $h(0,0)<\beta$ the unique solution $u$ of the equation is in $C^{m}(I)$.

4. Third-kind Volterra integral equations with weakly singular kernels. As in the previous section we start by looking at the properties of the operator

$$
\left(T_{k, \beta, \alpha} u\right)(t)=\int_{0}^{t} t^{-\beta}(t-x)^{-\alpha} k(t, x) u(x) d x,
$$

where now $0<\alpha<1$.

Theorem 4.1. For the values of $\alpha$ and $\beta$ described below the integral operator (4.1) has the following mapping properties:

(i) If $0<\alpha+\beta<1, T_{k, \beta, \alpha}$ is compact and bounded on $C(I)$.

(ii) If $\alpha+\beta=1$, the operator maps $C[0, T]$ into itself as a bounded operator. When $k(0,0)=0$ the operator is compact; otherwise, we have a non-compact operator whose spectrum is

$$
\sigma_{C[0, T]}\left(T_{k, \beta, \alpha}\right)=\{0\} \cup\{k(0,0) B(1+\lambda, 1-\alpha): \lambda \in \mathbb{C}, \operatorname{Re} \lambda \geq 0\} .
$$

Moreover, if $k \in C^{m}(\Delta), T_{k, \beta, \alpha}$ maps $C^{m}(I)$ to $C^{m}(I)$ and has the spectrum

$$
\begin{gathered}
\sigma_{C^{m}(I)}\left(T_{k, \beta, \alpha}\right)=\{0\} \cup\{k(0,0) B(1+\lambda, 1-\alpha): \lambda=0,1, \ldots, m-1\} \\
\cup \quad\{k(0,0) B(1+\lambda, 1-\alpha): \operatorname{Re}(\lambda) \geqslant m\} .
\end{gathered}
$$

Here, $B(\cdot, \cdot)$ is the Euler beta function.

Proof. We can write the operator $T_{k, \beta, \alpha}$ describing the third-kind Volterra integral equation (2.1) in the form

$$
T_{k, \beta, \alpha} u(t):=\int_{0}^{t} t^{-1} t^{1-\beta-\alpha}\left(1-\frac{x}{t}\right)^{-\alpha} k(t, x) u(x) d x .
$$


(i) If $0<\alpha+\beta<1$, the operator is compact, with the core $\varphi=(1-x)^{-\alpha} \in L^{1}(0,1)$ and $a(t, x):=t^{1-\beta-\alpha} k(t, x)$, where $a \in C(\Delta)$ and $a(0,0)=0$. The result then follows from [21].

(ii) If $\alpha+\beta=1$, the operator is cordial, with the core $\varphi(x)=$ $(1-x)^{-\alpha} \in L^{1}(0,1)$ and $a(t, x):=k(t, x)$.

The proof is complete.

We observe that, for $\alpha+\beta>1$, if the integral operator (4.1) is to be a bounded operator, the kernel must be of the form

$$
k(t, x)=x^{\alpha+\beta-1} h(t, x),
$$

where $h \in C(\Delta)$.

The following example illustrates this observation.

Example 4.2. Let $\beta=3 / 2, \alpha=1 / 2$ and $k(t, x) \equiv 1$. Then the operator

$$
T_{k, \beta, \alpha} u(t)=\int_{0}^{t} t^{-3 / 2}(t-x)^{-1 / 2} u(x) d x,
$$

maps the constant function $u(t) \equiv 1$ into $4 / t$ which is unbounded and non-continuous at $t=0$.

In the next theorem, we describe the mapping properties of the operator $T_{k, \beta, \alpha}$.

Theorem 4.3. Let $0<\alpha<1$ and $\alpha+\beta>1$, and assume that the kernel of the operator (4.1) has the form (4.4), where $h \in C(\Delta)$. Then it maps $C(I)$ boundedly into itself, and it is compact when $h(0,0)=0$. Otherwise, we have a non-compact operator with the spectrum

$$
\sigma_{C(I)}\left(T_{k, \beta, \alpha}\right)=\{0\} \cup\{h(0,0) B(\alpha+\beta+\lambda, 1-\alpha): \lambda \in \mathbb{C}, \operatorname{Re} \lambda \geq 0\} .
$$

Moreover, if $h \in C^{m}(\Delta)$, then $T_{k, \beta, \alpha}$ maps $C^{m}(I)$ to $C^{m}(I)$, and its spectrum is

$$
\begin{array}{r}
\sigma_{C^{m}(I)}\left(T_{k, \beta, \alpha}\right)=\{0\} \cup\{h(0,0) B(\alpha+\beta+\lambda, 1-\alpha): \lambda=0,1, \ldots, m-1\} \\
\cup\{h(0,0) B(\alpha+\beta+\lambda, 1-\alpha): \operatorname{Re}(\lambda) \geqslant m\} .
\end{array}
$$

Proof. Under the conditions of this theorem, the operator $T_{k, \beta, \alpha}$ is cordial: its core $\varphi(x)=x^{\alpha+\beta-1}(1-x)^{-\alpha}$ is in $L^{1}(0,1)$, and 
$a(t, x):=h(t, x)$ is continuous on $\Delta$. Theorem 2.1 of [21] then yields the desired result.

The following theorem provides necessary conditions for the thirdkind integral equation (1.1) with $0<\alpha<1$ to have a unique continuous solution. In the case where $\alpha+\beta>1$, the kernel is assumed to be of the form (4.4), with $h \in C(\Delta)$.

Theorem 4.4. Consider the third-kind Volterra integral equation (1.1) with $0<\alpha<1, k \in C(\Delta)$ and $f \in C(I)$ such that $g(t):=t^{-\beta} f(t)$ is continuous on $I$. Then the following statements are true:

(a) If $0<\alpha+\beta<1$, there exists a unique continuous solution whenever $g \in C(I)$.

(b) If $\alpha+\beta=1$ and if $1 \in \sigma_{C[0, T]}\left(T_{k, \beta, \alpha}\right)$, where

$$
\sigma_{C[0, T]}\left(T_{k, \beta, \alpha}\right)=\{0\} \bigcup\{k(0,0) B(1+\lambda, 1-\alpha): \lambda \in \mathbb{C}, \operatorname{Re} \lambda \geq 0\},
$$

the equation does in general not have a unique solution: it may either have at most one continuous solution, or it has multiple solutions.

For $1 \notin \sigma_{C[0, T]}\left(T_{k, \beta, \alpha}\right)$, the equation has a unique continuous solution for all $g \in C(I)$.

(c) Let $\alpha+\beta>1$ and the kernel be of the form (4.4) with $h \in C(\Delta)$ : If $1 \in \sigma_{C[0, T]}\left(T_{k, \beta, \alpha}\right)$, where

$\sigma_{C[0, T]}\left(T_{k, \beta, \alpha}\right)=\{0\} \cup\{h(0,0) B(\lambda+\alpha+\beta, 1-\alpha): \lambda \in \mathbb{C}, \operatorname{Re} \lambda \geq 0\}$,

the equation does in general not possess a unique continuous solution: it may either have at most one continuous solution, or it has multiple continuous solutions.

For $1 \notin \sigma_{C[0, T]}\left(T_{k, \beta, \alpha}\right)$, the equation has a unique continuous solution.

Remark 4.5. For more details on (b) and (c), see [23].

The next two theorems exhibit the regularity properties of the solution of the third-kind Volterra integral equation (1.1) for $0<\alpha<1$ and $\beta+\alpha \geq 1$. 
Theorem 4.6. Let $\beta+\alpha=1$, and assume that $k \in C^{m}(\Delta)$ and $g \in C^{m}(I)$. Then, for $1 \notin \sigma_{C[0, T]}\left(T_{k, \beta, \alpha}\right)$, the unique solution $u \in C(I)$ of the equation (1.1) is in $C^{m}(I)$.

Theorem 4.7. Assume that $\beta+\alpha>1$, and let $h \in C^{m}(\Delta)$ and $g \in C^{m}(I)$. Then, for $1 \notin \sigma_{C[0, T]}\left(T_{k, \beta, \alpha}\right)$, the unique solution $u \in C(I)$ of the equation (1.1) is in $C^{m}(I)$.

Remark 4.8. In [22], von Wolferdorf considered the special third-kind Volterra integral equation

$$
x^{\beta} \omega(x)=\gamma \int_{0}^{x}(x-\xi)^{\beta-1} \omega(\xi) d \xi+h(x), \quad \beta>0,
$$

where $\gamma$ denotes a positive parameter. He studied the existence solutions for (4.6) that are of the form $\omega(x)=x^{\sigma} Z(x)$ where $Z(x)$ is the solution of the third-kind equation

$$
x^{\beta+\sigma} Z(x)=\gamma \int_{0}^{x}(x-\xi)^{\beta-1} \xi^{\sigma} Z(\xi) d \xi+h(x) .
$$

Here, $\sigma$ is the real root of the equation

$$
\gamma B(\eta, \sigma+1)=1 .
$$

Based on the resolvent representation of the solution of (4.7) for an arbitrary $\beta>0$, he established a sufficient condition for the solvability of the third-kind Volterra integral equation (4.6).

Comparison of the equations (4.6) and (4.7) with the equation (1.1) reveals that the results on the existence and uniqueness of the solution follow from the theory discussed in the present paper. Consider first the equation (4.6):

(a) If $0<\beta<1$, von Wolfersdorf's result follows from Theorem 4.4.

(b) If $\beta=1$, Theorem 3.2 furnishes von Wolfersdorf's result.

(c) In the case $\beta>1$, the solvability result is obtained similar to (a).

Regarding the existence and uniqueness of $Z(x)$ in (4.7) we have the following:

(a) For $0<\beta<1$, Theorem 4.4 will give the appropriate result.

(b) If $\beta=1$, von Wolfersdorf's result follows from Theorem 3.11.

(c) Finally, if $\beta>1$, the solvability result is found similar to case (a). 
5. Concluding remarks and future work. As we observed in Section 3, for the case $\beta>1$, we need the kernel to be of the form (3.4) in order to have a bounded operator. The case $h \in C(\Delta)$ was explained in that section.

The more interesting case arises when $k \in C(\Delta)$ but $h$ is not continuous with respect to $x$. We will show that, under some weaker conditions on $h$, the operator is still bounded as an operator on $C(I)$. Since this case is not covered by Vainikko's theory of cordial Volterra integral operators, we need to establish the properties of the operator $T_{k, \beta}$ separately. The following examples are meant to illustrate this statement.

Example 5.1. Let the kernel in the operator $T_{k, \beta}$ be

$$
k(t, x)= \begin{cases}\frac{x^{\beta-1} \sin (1 / x)}{-\ln (t)} & \text { if }(t, x) \neq(0,0) \\ 0 & \text { if }(t, x)=(0,0)\end{cases}
$$

It is clear that $k \in C(\Delta)$, where $\Delta=\{(t, x): 0 \leq x \leq t \leq T\}$, and $T<1$, but

$$
h(t, x)=\frac{\sin (1 / x)}{-\ln (t)}
$$

is not continuous at $x=0$. So, if we write our operator in the form of (3.6), with $h(t, x)$ not continuous at $x=0$, it follows that the theory of cordial operators is not applicable. However, this operator maps $C(I)$ into itself. It is a bounded operator and it is also compact.

Example 5.2. Let the kernel in the operator $T_{k, \beta}$ be

$$
k(t, x)= \begin{cases}\frac{x^{\beta-1} \ln (x)}{-\ln (t)}, & \text { if }(t, x) \neq(0,0) \\ 0, & \text { if }(t, x)=(0,0) .\end{cases}
$$

It is clear that, similarly to the previous example, if $k \in C(\Delta)$ and $T<1$, but $h(t, x)=\ln (x) /-\ln (t)$ is not continuous at $x=0$, the theory of cordial Volterra integral operators again is not applicable in this case. This operator maps $C(I)$ into itself, is bounded and noncompact, and its spectrum is given by

$$
\sigma\left(T_{k, \beta, \alpha}\right)=\left\{\frac{1}{\beta+\lambda} \mid \lambda \in \mathbb{C}, \operatorname{Re} \lambda \geq 0\right\} .
$$


Remark 5.3. According to Example 5.2, we can say in general that the integral operator of the form

$$
\int_{0}^{t} t^{-1}\left(\sum_{i=1}^{n} a_{i}(t) \varphi_{i}(s / t)\right) u(s) d s,
$$

where the $a_{i}(t)$ are continuous functions at least in a neighborhood of $t=0(i=1, \ldots, n)$ and $\varphi_{i}(x) \in L^{1}(0,1)$, is a linear combination of cordial operators but it is not a cordial operator.

The general theory underlying the above examples is currently being established and will be presented soon.

We conclude this section by noting that the regularity results presented in this paper will form the basis of the convergence analysis for piecewise polynomial collocation solutions to Volterra integral equations of the form (1.1).

Acknowledgments. The authors gratefully acknowledge the detailed and constructive comments by the two referees; they led to a significant improvement in the presentation of our results.

The first author also would like to thank Dr. Maria Teresa Diogo for her valuable discussions and scientific suggestions. Most of S. Seyed Allaei's research was carried out while she visited the Department of Mathematics at HKBU (Prof. Hermann Brunner and Prof. Tao Tang) from October 2013 to April 2015.

\section{REFERENCES}

1. G.R. Bart and R.L. Warnock, Linear integral equations of the third kind, SIAM J. Math. Anal. 4 (1973), 609-622.

2. H. Brunner, Collocation methods for Volterra integral and related functional equations, Cambridge University Press, Cambridge, 2004.

3. H.G. Bžihatlov, A certain integral equation of the third kind, Izv. Akad. Nauk. 2 (1970), 18-23.

4. A.R. Chvoles, On Fredholm's integral equations of the third kind, Bull. Acad. Sci. Georgian 2 (1941), 389-395.

5. G.C. Evans, Volterra's integral equation of the second kind with discontinuous kernel, Trans. Amer. Math. Soc. 11 (1910), 393-413.

6. Volterra's integral equation of the second kind with discontinuous kernel II, Trans. Amer. Math. Soc. 11 (1911), 429-472. 
7. T. Fényes, On the operational solution of a convolution type integral equation of the third kind, Stud. Sci. Math. Hungar. 12 (1977), 65-75.

8. N.S. Gabbasov, On the theory of Fredholm integral equations of the third kind in a space of generalized functions, Izv. Vyssh. Uchebn. Zaved. Mat. 85 (1986), 6870 .

9. - Approximate solution of integral equations of the third kind, Izv. Vyssh. Uchebn. Zaved. Mat. 82 (1986), 49-52.

10. N.S. Gabbasov and S.A. Solov'eva, Special versions of the collocation method for a class of integral equations of the third kind, Russ. Math. 56 (2012), 22-27.

11. P. Grandits, A regularity theorem for a Volterra integral equation of the third kind, J. Integral Equations Appl. 20 (2008), 507-526.

12. D. Hilbert, Grundzüge einer allgemeinen Theorie der linearen Integralgleichungen, Teubner, Leipzig, 1912.

13. M.I. Imanaliev and A. Asanov, Regularization and uniqueness of solutions of systems of nonlinear Volterra integral equations of the third kind, Dokl. Math. 76 (2007), 490-493.

14. N.N. Juhanonov, General theorems on the solvability of a certain convolution type singular integral equation of the third kind on the half-axis, Izv. Akad. Nauk. 3 (1973), 22-28.

15. S.V. Pereverzev and S.A. Prössdorf, Discretization of Volterra integral equations of the third kind with weakly singular kernels, J. Inv. Ill-Posed Prob. 5 (1997), 565-577.

16. É. Picard, Sur les équations intégrales de troisième espèce, Ann. Sci. École Norm. 28 (1911), 459-472.

17. V.S. Rogožin and S.N. Raslambekov, The Noether theory for integral equations of the third kind in spaces of continuous and generalized functions, Sov. Math. 23 (1979), 48-53.

18. T. Sato, Sur l'équation intégrale $x u(x)=f(x)+\int_{0}^{x} K(x, t, u(t)) d t$, J. Math. Soc. Japan 5 (1953), 145-153.

19. E. Schock, Integral equations of the third kind, Stud. Math. 81 (1985), 1-11.

20. G. Vainikko, Cordial Volterra integral equations 1, Numer. Funct. Anal. Optim. 30 (2009), 1145-1172.

21. Cordial Volterra integral equations 2, Numer. Funct. Anal. Optim. 31 (2010), 191-219.

22. L.V. Wolfersdorf, On the theory of convolution equations of the third kind, J. Math. Anal. Appl. 331 (2007), 1314-1336; 342 (2008), 838-863.

23. Z.W. Yang, Second-kind linear Volterra integral equations with noncompact operators, Numer. Funct. Anal. Optim. 36 (2015), 104-131. 
Cemat-Departamento de Matemática, Instituto Superior Técnico, UL, Lisbon, Portugal

Email address: sonia.seyedallaei@tecnico.ulisboa.pt

Department of Mathematics, Harbin Institute of Technology, Harbin 150001, China

Email address: yangzhan_wen@126.com

Department of Mathematics, Hong Kong Baptist University, Kowloon Tong, Hong Kong SAR, China and Department of Mathematics and Statistics, Memorial University of Newfoundland, St. John's, NL, Canada A1C 5S7

Email address: hbrunner@math.hkbu.edu.hk 